\title{
FORCE CONTROL OF PNEUMATIC SERVO SYSTEM USING NEURAL NETWORK
}

\author{
Toshiro NORITSUGU and Kosuke FUKUZONO \\ Faculty of Engineering, Okayama University \\ 3-1-1 Tsushimanaka, Okayama, 700 Japan
}

\begin{abstract}
A pneumatic servo system has been expected to be applied to contact tasks because of the ability of collision force absorption, delicate force control and so on due to air compressibility. However, the high nonlinearity and friction in the pneumatic system make a satisfactory servo-type force control not easy. The purpose of this study is to develop a pneumatic servo-type force control system which can be applied to the system with unknown and variable parameters and environments by using a neural network controller with the capability of learning and adaptability.

The controller is installed in parallel with an usual linear controller. Some experiments confirm that the neural network controller is one of effective approaches to realize the advanced force control of a pneumatic servo system.
\end{abstract}

\section{KEY WORDS}

Pneumatic servo, Force control, Neural network control, Learning, Adaptability

\section{INTRODUCTION}

A pneumatic actuation has been used mainly for simple tasks. Recently, however, it is desired to develop the pneumatic servo system with more elaborate control performance to be applied to robotics and mechatronics. A contact task is one of the effective applications of a pneumatic servo system because of the flexibility from air compressibility which produces the important ability of collision force absorption, delicate force control and so on. The fine control performance can be obtained by some control strategies of an optimal control and an adaptive control and so on [1]. However, the design of such a linear controller requires mathematical modelling and identification of a controlled plant, which are not necessarily easy in the pneumatic system. Also the property of the contacted object is not always exactly known and constant. A neural network controller is useful for such a kind of plant.

In this study, a neural network is applied to the force control of a servo system comprising a pneumatic cylinder and electro-pneumatic proportional control valves. The neural network with input/output layers 
and one hidden layer is installed in parallel with the usual linear PI controller. The network learns the dynamics of the controlled pneumatic plant including the object based on the back propagation algorithm referring to the reference input signal, the output signal and their derivatives. The control error is used as a teaching signal, which is made minimum through the learning. First, the proposed control system using a fundamental neural network is described. Next, its ability of both learning and adaptability for the change of the object stiffness are discussed experimentally.

Finally, an improved learning algorithm considering the plant dynamics is proposed. This study assures the effectiveness of the neural network control to realize an advanced pneumatic servo-type force control system.

\section{CONTROL SYSTEM}

\section{Structure of control system}

Figure 1 shows the proposed control system. A control input $\boldsymbol{u}$ is the sum of outputs $u_{f}$ and $u_{N}$, from feedback $\left(\mathrm{C}_{f}\right)$ and neural network $(\mathrm{NN})$ controllers, respectively. $\mathrm{C}_{\mathrm{f}}$ contributes to ensuring the stability of the system at the beginning of learning. NN adds the adaptability for variations of operational conditions. With the progress of learning, the output from linear controller decreases and the neural network controller becomes to dominate the overall control system. The control error $e\left(=f_{r}-f\right)$ is used as a teaching signal to be minimized.

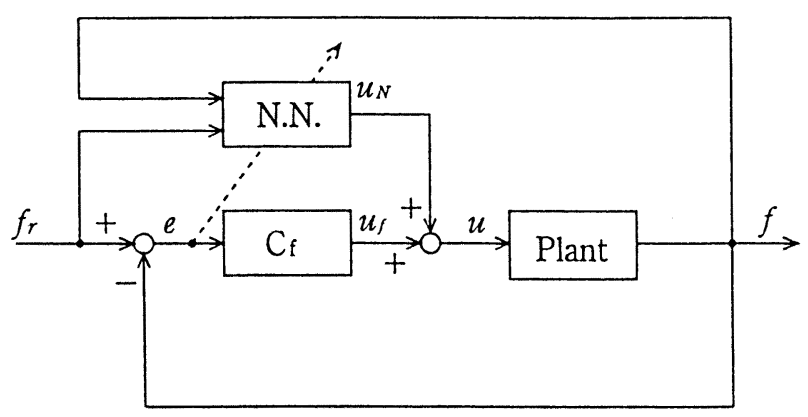

Figure 1. Proposed neural network control system

\section{Neural network}

Figure 2 shows the neural network built up in the personal computer with $\mathrm{C}$ program language. The input layer has seven neurons including a neuron with the output of -1 to set the bias value of each neuron in the hidden layer. There are fourteen neurons including a neuron with -1 in the hidden layer. All layers are connected in only the forward direction. The input to each neuron is given as the weighted sum of outputs from the previous layer. The output of each neuron is generated by the linear function in the input layer, in the hidden and output layers the following sigmoid function is used.

$$
f_{s i g}(x)=\frac{1}{1+\exp (-x)}
$$

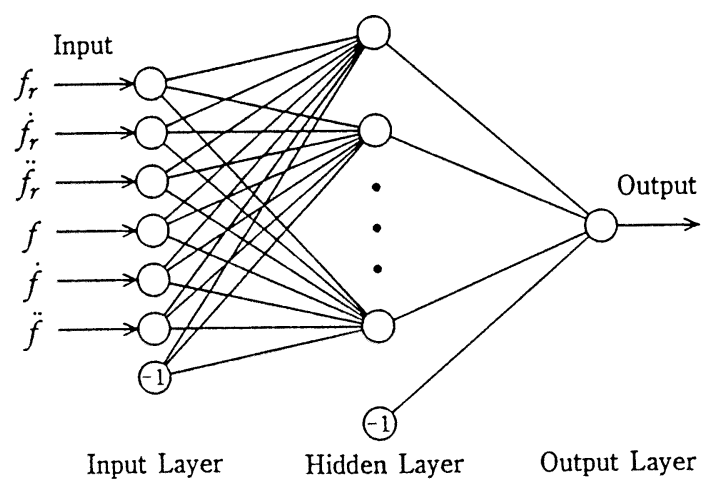

Figure 2. Structure of neural network

\section{Leaming algorithm}

In Figure 2, the following symbols are defined:

$i_{j}^{I}$ : input to the $j$ th neuron in the input layer

$o_{. j}^{I}$ : output from the $j$ th neuron in the input layer

$i_{k}^{\prime \prime}$ : input to the $k$ th neuron in the hidden layer

$o_{k}^{\prime \prime}$ : output from the $k$ th neuron in the hidden layer

$i^{O}$ : input to the output layer

$o^{O}$ : output from the output layer

$w_{j k}^{l H}:$ weight from the $j$ th neuron in the input layer to the $k$ th neuron in the hidden layer

$w_{k}^{H O}$ : weight from the $k$ th neuron in the hidden layer to the output layer

The control input $u_{N}$ is given by

$$
\boldsymbol{u}_{N}=\boldsymbol{k}_{N}\left(\boldsymbol{o}^{o}-0.5\right)
$$

The operation of each neuron is described as:

$$
\begin{aligned}
& o_{j}^{l}=i_{j}^{l} \\
& o_{k}^{H}=f_{s i g}\left(i_{k}^{H}\right), i_{k}^{H}=\sum_{j} w_{j k}^{I H} o_{j}^{l} \\
& o^{O}=f_{s i g}\left(i^{O}\right), i^{O}=\sum_{k} w_{k}^{H O} o_{K}^{H}
\end{aligned}
$$

The learning process is based on the back propagation algorithm, which minimizes $E$ given by

$$
E=\frac{1}{2} e^{2}
$$


The weights are updated by the following increments to minimize $E$ :

$$
\begin{aligned}
& \Delta w_{j k}^{I H}=-\eta^{+} \frac{\partial E}{\partial w_{j k}^{I I I}} \\
& \Delta w_{k}^{H O}=-\eta^{+} \frac{\partial E}{\partial w_{k}^{H O}}
\end{aligned}
$$

where $n^{*}>0$ is a learning rate to determine the speed of learnifing.

$\partial E / \partial w_{k}^{H O}$ in Eq.(8) can be calculated by

$$
\begin{aligned}
\frac{\partial E}{\partial w_{k}^{H O}} & =\frac{\partial E}{\partial i^{O}} \frac{\partial i^{O}}{\partial w_{k}^{H O}} \\
\frac{\partial i^{O}}{\partial w_{k}^{H O}} & =\frac{\partial}{\partial w_{k}^{H O}}\left(\sum_{k} w_{k}^{H O} o_{k}^{I I}\right)=o_{k}^{H I} \\
\frac{\partial E}{\partial i^{O}} & =-\delta^{O *} \\
\frac{\partial E}{\partial w_{k}^{H O}} & =-\delta^{O *} \boldsymbol{o}_{k}^{H}
\end{aligned}
$$

where $\delta^{\circ *}$ is called a generalized error calculated by

$$
\begin{aligned}
& \delta^{O *}=-\frac{\partial E}{\partial f} \frac{\partial f}{\partial o^{O}} \frac{\partial o^{O}}{\partial i^{O}} \\
& \frac{\partial E}{\partial f}=\frac{\partial}{\partial f}\left\{\frac{1}{2}\left(f_{r}-f\right)^{2}\right\}=-e \\
& \frac{\partial o^{O}}{\partial i^{O}}=\frac{\partial f_{s i g}\left(i^{O}\right)}{\partial i^{O}}=f_{s i g}^{\prime}\left(i^{O}\right)
\end{aligned}
$$

The dynamics of the controlled plant is not considered to calculate $\partial f / \partial o^{O}$ assumed to be constant.

$$
\frac{\partial f}{\partial o^{o}}=a_{o}
$$

The increment of weight can be written as:

$$
\Delta w_{k}^{H O}=\eta^{*} \delta^{O *} o_{k}^{H}
$$

Defining the modified generalized error $\delta^{\circ}=\delta^{\circ *} / a$ o and the learning rate $\eta=\eta^{*} a o$, the number of parameters to be set can be decreased. Consequently, the weight is updated by

$$
w_{\mathrm{k}}^{\mathrm{HO}}=w_{\mathrm{k}}^{\mathrm{HO}}+\eta \delta^{\mathrm{O}} o_{\mathrm{k}}^{\mathrm{H}}
$$

The update equation Eq.(19) of the weight $w_{j k}^{l H}$ can be derived in the same manner.

$$
w_{. j k}^{I H}=w_{j k}^{I H}+\eta \delta_{k}^{I I} \boldsymbol{o}_{. i}^{\prime}
$$

\section{EXPERIMEINTAL PNEUMATIC SERVO SYSTEM}

Figure 3 shows the pneumatic servo system. A single rod pneumatic cylinder with inner diameter of $50 \mathrm{~mm}$, rod diameter of $20 \mathrm{~mm}$ and full stroke of $200 \mathrm{~mm}$ is horizontally installed, which is connected with a load mass of $26.5 \mathrm{~kg}$ sliding on a guide rail. The supply pressure $P s$ is $490 \mathrm{kPa}$. The mass contacts with the object through a force sensor. Four proportional flow control valves operate as a single input four way control valve, of which the change of the opening areas from the equilibrium state $(f=0)$ are adjusted as a control input $u$. The contact force $f$ is detected with the resolution of $0.51 \mathrm{~N}$, of which derivatives are computed by the differential approximation. The control program is written with $\mathrm{C}$ language.

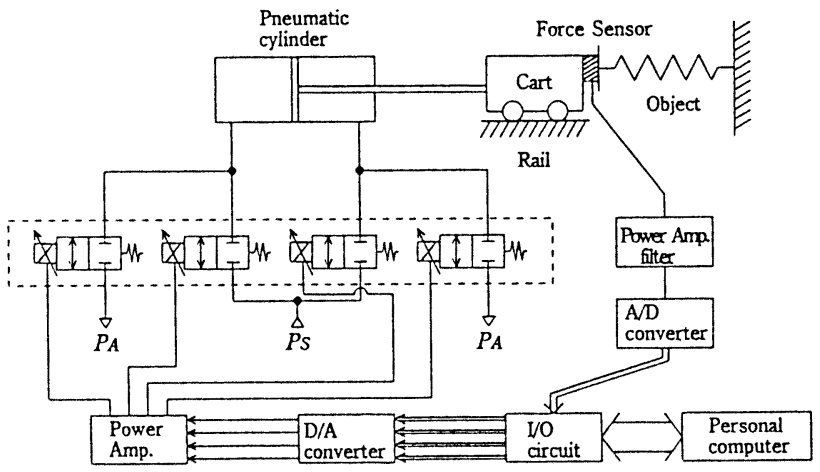

Figure 3. Experimental pneumatic servo system

\section{EXPERIMENTAL RESULTS AND DISCUSSION}

A steel piece and a spring with stiffness of $3900 \mathrm{~N} / \mathrm{m}$ are used as an object. For two objects, the control performances from an usual PI controller with constant gains and the proposed neural network controller are discussed. The discrete-time control input from the PI controller is given by

$$
\begin{aligned}
& u(k)=k_{P} e(k)+k_{I} \sum_{k} e(k) T_{s} \\
& e(k)=f_{I}(k)-f(k)
\end{aligned}
$$

$T_{s}$ is the sampling period of $1 \mathrm{~ms}$. It is $2.6 \mathrm{~ms}$ in the 
neural network control system. The initial values of weights of the network are given by random numbers from -0.1 to 0.1 . In Eq.(2), $k_{N}=1.0 \times 10^{-6}$.

\section{Adaptability to object stiffness}

Figure 4 shows the response of the PI control system with control gains optimally adjusted in an experiment for the rigid object, i.e., $k p=1.0 \times 10^{-8} \mathrm{~m}^{2} / \mathrm{N}, k l=1.0$ $\times 10^{-8} \mathrm{~m}^{2} / \mathrm{N} \cdot \mathrm{s}$. (a) is the response for the rigid object. It follows nearly the reference, but not a little the phase lag remains, which cannot be perfectly compensated by the PI controller. The same PI controller gives the response in (b) for the elastic object. The change of the object stiffness varies the property of the controlled plant, so that the response becomes worse. The control gains are readjusted $t$ for the elastic object as $k p=1.5 \times$ $10^{-8} \mathrm{~m}^{2} / \mathrm{N}, k i=2.0 \times 10^{-8} \mathrm{~m}^{2} / \mathrm{N} \cdot \mathrm{s}$. Although the response can be improved to some extent, the phase lag still remains, also a stick-slip phenomena appears due to the static friction as shown in Figure 5. The usual PI controller with constant gains cannot sufficiently deal with the variation of the plant parameters including the stiffness of the object.



(a) Rigid object

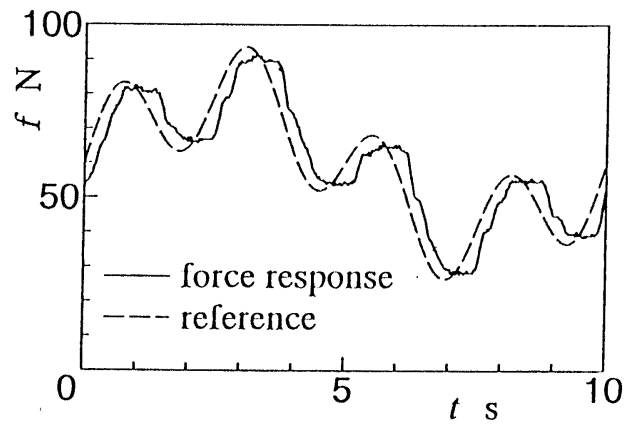

(b) Elastic object

Figure 4. Usual PI control

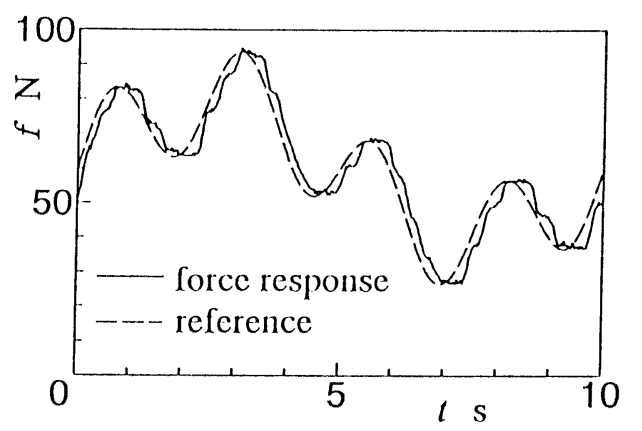

Figure 5. Readjusted usual PI control for elastic object

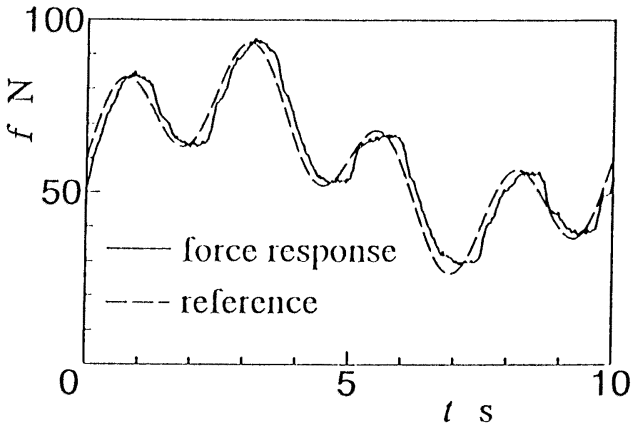

(a) Beginning of learning

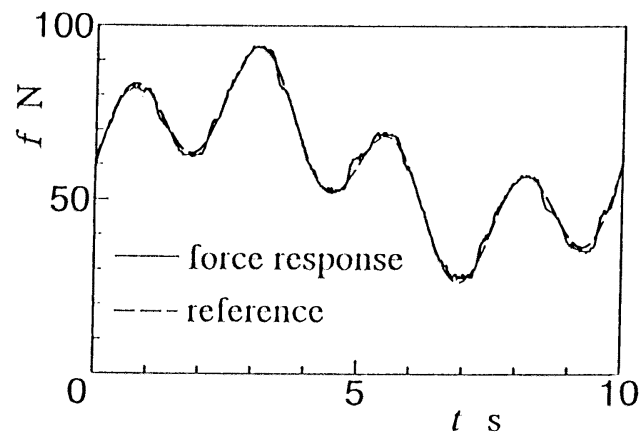

(b) End of learning

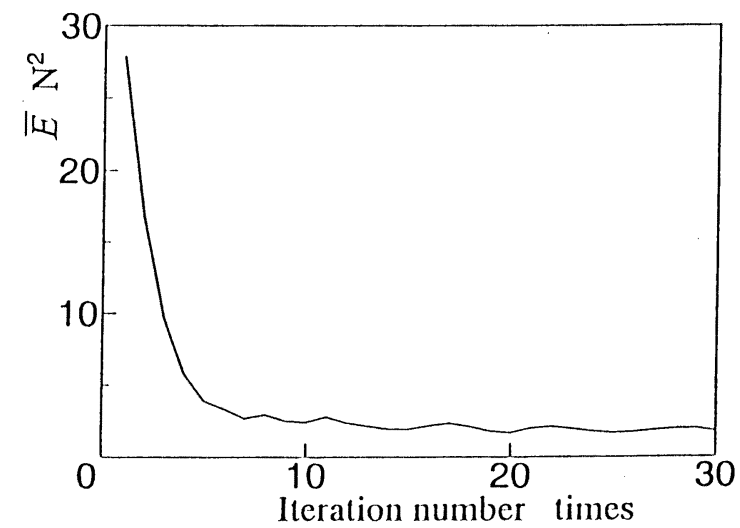

(c) Transition of performance index

Figure 6. Neural network control for elastic object 
Figure 6 shows the result of the neural network control system shown in Figure 1 for the elastic object. The learning rate $\eta$ is $2.5 \times 10^{-5}$. The PI controller in Figure 4(a) and (b) is used as a feedback controller $\mathrm{C}_{\mathrm{f}}$.

(a) and (b) are the response at the beginning and after the end (at the 30th trial) of learning, respectively.

Where one trial corresponds to one period (10 seconds) of the reference input. (c) shows the transition of the performance index defined by

$$
\begin{aligned}
& \bar{E}=\frac{2 J}{N_{S}} \\
& J=\sum_{\phi} \frac{1}{2} e^{2}
\end{aligned}
$$

where $J$ is the sum of sampled values in one trial, $N s$ means the sampling times over one trial.

To examine the adaptability for the change of stiffness of the object, the initial weights of the network are set to the values enough learned for the rigid object. As shown in Figure 6(a), the control error is caused by the variation of the plant dynamics due to the change of stiffness at the first trial. The error can be decreased by the learning to obtain the response in Figure 6(b), which is nearly the same as the response for the rigid object being left out on account of limited space. The comparison of Figure 6 (b) with Figure 4(b) and 5 can clarify the effectiveness of the neural network control. Especially, the ability of the neural network controller to compensate the influence of the nonlinear friction is interested. Also the learning process can converge enough fast for the practical application.

\section{Effect of linear feedback controller}

Figure 7 shows the effect of the linear feedback controller $\left(\mathrm{C}_{n}\right.$ on the learning performance examined experimentally for the elastic object with the same reference input as before. The neural network control system with the PI controller same as in Figure 6 or P controller with $k p=1.0 \times 10^{-8} \mathrm{~m}^{2} / \mathrm{N}$ can converge very fast, where $\eta=1.0 \times 10^{-3}$. When only the neural network controller is used, the learning process becomes unstable for $\eta=1.0 \times 10^{-3}$. For $\eta=5.0 \times$ $10^{-4}$, it can converge but requires many trials until the satisfactory control accuracy is realized. $\mathrm{Cf}_{\mathrm{f}}$ can stabilize the learning process not only at the beginning but also during the learning, so that the higher learning rate can be set to make the convergence fast. Since the important role of $\mathrm{C}_{\mathrm{f}}$ is only to improve the stability of the learning process, the exactly gain tuning is not required. The control accuracy can be mainly ensured by the neural network controller.

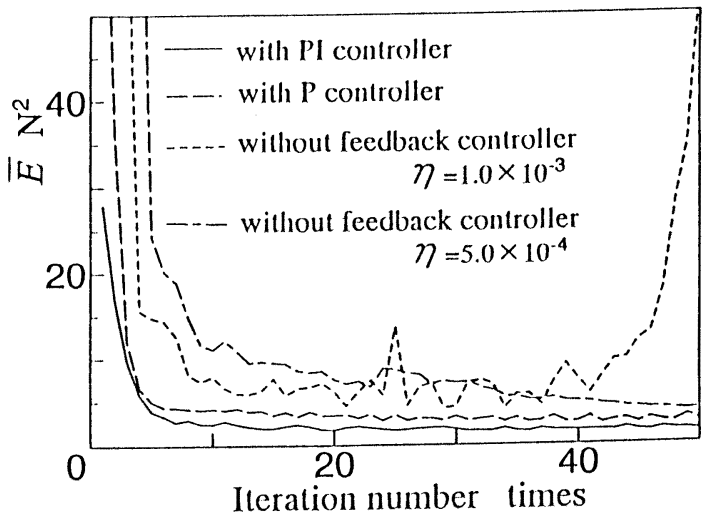

Figure 7. Effect of linear feedback control on learning

\section{LEARNING CONSIDERING PLANT DYNAMICS}

Influence of reference input frequency

Figure 8 shows the responses at the 80th learning trial of the control system with the same PI controller as the above when the sinusoidal reference input with $0.3 \mathrm{~Hz}$ and $1 \mathrm{~Hz}$ is commanded for the elastic object. The response can well follow the reference with $0.3 \mathrm{~Hz}$, but not well for $1 \mathrm{~Hz}$ where the large control error remains. It seems the reason for the deterioration of the control performance at $1 \mathrm{~Hz}$ to disregard the plant dynamics to calculate Eq.(16) in deriving the learning algorithm.

Improved learning algorithm

The plant dynamics is described by the following discrete-time equation.

$$
f=\sum_{m} a_{m}\left(u_{N}\right)_{n-m}
$$

where the subscript $(n-m)$ means the value before $m$ samplings from nth sampling. $a m(m=1, \cdots, M)$ denote plant parameters. Also, Eqs.(7), (8) are written as:

$$
\begin{aligned}
& \Delta w_{j k}^{I H}=-\eta \sum_{m}^{M} \frac{\partial E}{\partial\left(w_{j k}^{H H}\right)_{n-m}} \\
& \Delta w_{k}^{H O}=-\eta \sum_{m}^{M} \frac{\partial E}{\partial\left(w_{k}^{H O}\right)_{n-m}}
\end{aligned}
$$

Considering the above plant dynamics, the following expression can be derived in the same manner as Eqs.(18), (19).

$$
w_{j k}^{I H}=w_{. j k}^{I H}+\eta \sum_{m}^{M}\left(\delta_{k}^{H}\right)_{n-m}\left(o_{j}^{l}\right)_{n-m}
$$


$w_{\mathrm{k}}^{\mathrm{HO}}=w_{\mathrm{k}}^{\mathrm{HO}}+\eta \sum_{m}^{M}\left(\delta^{\circ}\right)_{n-m}\left(\boldsymbol{O}_{\mathrm{k}}^{\mathrm{H}}\right)_{n-m}$

where,

$$
\begin{aligned}
\left(\delta_{k}^{H}\right)_{n-m}=\left(\delta^{O}\right)_{n-m}\left(w_{k}^{H O}\right)_{n-m} \\
f_{\text {si } g}^{\prime}\left(\left(i_{k}^{H}\right)_{n-m}\right) \\
\left(\delta^{O}\right)_{n-m}=\boldsymbol{e} \boldsymbol{a}_{m} k_{N} f_{\text {sig }}^{\prime}\left(\left(i^{O}\right)_{n-m}\right)
\end{aligned}
$$

Figure 9 shows the response of the improved control system using Eqs.(27), (28) instead of Eqs.(18), (19) in the control system used in Figure 8. Where, $n=2.5$, $M=40$ and $T s=32.81 \mathrm{~ms}$. Comparing Figure 9 with Figure 8, the effect of the improved algorithm is remarkable. Also the convergence of the learning can be made faster by considering the plant dynamics in the learning algorithm.

$a_{n}$ in Eq.(24) must be known beforehand. Naturally, it is sufficient to calculate them using the nominal plant parameters. However, that cannot help injuring the important advantage of the neural network control what no mathematical plant model is required to design the controller. Some studies have discussed this problem [2,3]. Then, the proposed improved learning algorithm is available when the nominal plant model can be obtained relatively easily.

\section{CONCLUSIONS}

The neural network controller with an usual linear PI controller in parallel was proposed for the force control of a pneumatic servo system. The learning algorithm disregarding the plant dynamics is enough sufficient for reference input with relatively low frequency, which can accommodate to the unknown plant and object. If the nominal plant model can be obtained without much difficulties, the improved learning algorithm considering the plant dynamics is effective for the high frequency. The results can contribute to construct the advanced pneumatic servo-type force control system being of much practical use.

\section{REFERENCES}

1. Noritsugu, T., et al., Impedance Control of a Pneumatic Servo System with Adaptive Control Method, Journal of Robotics and Mechatronics, 1991, 3-6, pp.463-69.

2. Jordan, M.I., Generic Constraints on Underspecified Target Trajectories, Proceedings of IJCNN'89, Washington, D.C., 1989, pp.1217-25.

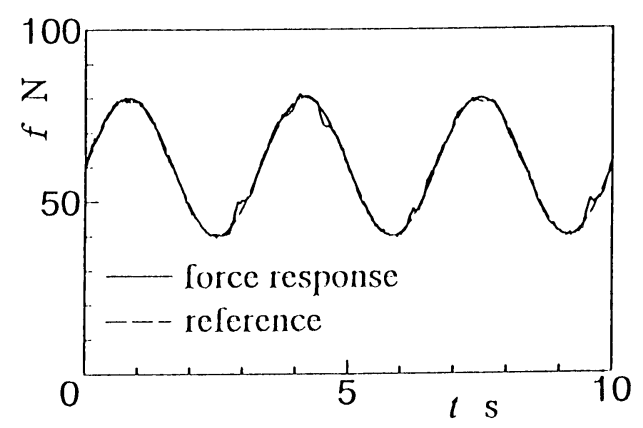

(a) Sinusoidal reference input with $0.3 \mathrm{~Hz}$

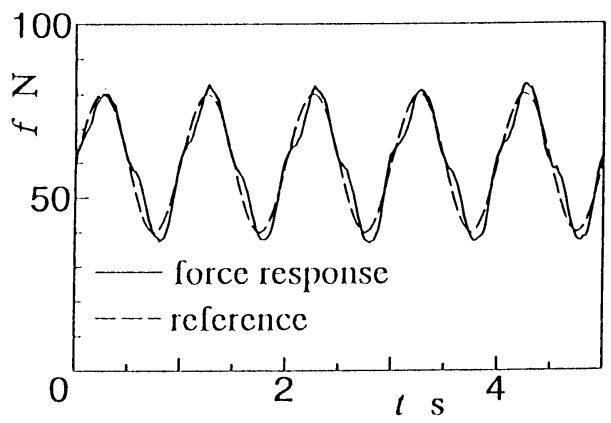

(b) Sinusoidal reference input with $1 \mathrm{~Hz}$

Figure 8. Learning property for fast reference input

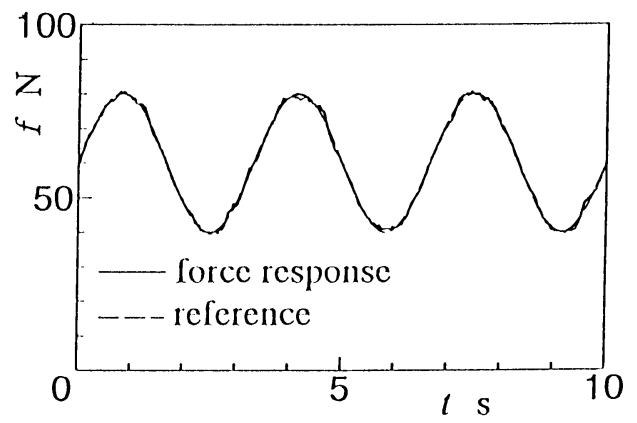

(a) Sinusoidal reference input with $0.3 \mathrm{~Hz}$

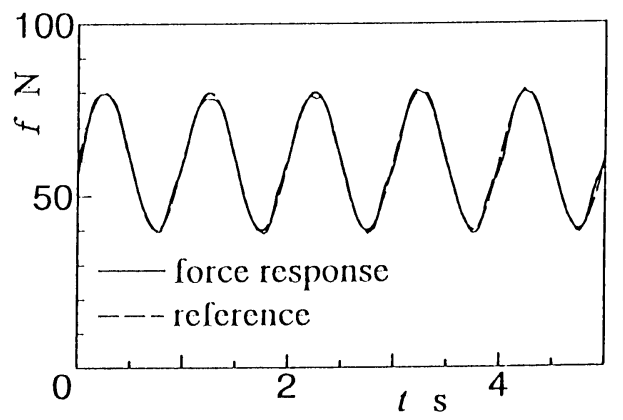

(b) Sinusoidal reference input with $1 \mathrm{~Hz}$

Figure 9. Learning property of improved algorithm 